\title{
Cerebral white matter lesions are not associated with apoE genotype but with age and female sex in Alzheimer's disease
}

\author{
Hideyuki Sawada, Fukashi Udaka, Yuishin Izumi, Kazuto Nishinaka, Hideshi Kawakami,
} Shigenobu Nakamura, Masakuni Kameyama

\begin{abstract}
Cerebral white matter lesions, such as leukoaraiosis, may be a result of damage from cerebral ischaemia, and may also be associated with the degenerative process in Alzheimer's disease. The apolipoprotein $\varepsilon 4$ (apo\&4) genotype is a genetic risk factor for both Alzheimer's disease and ischaemic brain damage through acceleration of atherosclerosis. The aim was to determine whether apos 4 may be related to the formation of cerebral white matter lesions in Alzheimer's disease. The association of apoE genotype, sex, age, and the presence of several vascular risk factors, with the presence of white matter lesions in 55 patients clinically diagnosed with Alzheimer's disease was investigated. The cerebral white matter lesions were identified by T2 weighted MRI and classified on a 4 grade scale from no lesion to diffuse lesion. The odds ratio (OR) of the factors mentioned above to the presence of white matter lesions was determined and tested by Fisher's exact test. The association of the lesion grades with these factors was analysed by non-parametric tests. The apoE 4 genotype was strongly associated with Alzheimer's disease $(p=0.0001)$, but not associated with the presence or the degree of cerebral white matter lesions in Alzheimer's disease $(O R=1.09, p>0.99)$. Aging ( $>70$ years old) was a significant risk factor for white matter lesions $(\mathrm{OR}=7.2, \mathrm{p}=0.0006)$ and age was significantly correlated with the lesion $(p=0.0075)$. The OR of female sex to the lesion grades was $2.89(p=0.084)$ and the lesion grade of female sex was significantly higher than that of the male sex $(p=0.047)$. Other vascular risk factors were not significantly associated with the presence of white matter lesions. These findings suggest that there is a sex difference in white matter pathology in Alzheimer's disease.
\end{abstract}

(F Neurol Neurosurg Psychiatry 2000;68:653-656)

Keywords: leukoaraiosis; Alzheimer's disease; apolipoprotein $\mathrm{E}$
It has been shown that cerebral white matter lesions, such as leukoaraiosis, can play an important part in cognitive disturbance in Alzheimer's disease. ${ }^{1}$ The pathogenetic mechanism of cerebral white matter lesions has not been resolved, but several factors, such as gliosis, demyelination, and ischaemic damage have been proposed as candidates. ${ }^{23}$ Leukoaraiosis in Alzheimer's disease is not a mere secondary Wallerian degeneration caused by neuronal loss in the cerebral cortex, because it is independent of the severity of the cerebral cortical lesions. ${ }^{2}$ Apolipoprotein $\mathrm{E}$ (apoE) is synthesised mainly in the liver and is a very low density lipoprotein. There are three major isoforms, E2, E3, and E4. Presence of the E4 isoform is linked to hyperlipidaemia ${ }^{4}$ and is a significant risk factor for vascular disorders, including fatal myocardial infarction. ${ }^{56} \mathrm{Al}-$ though the association between E4 and cerebral infarction has been investigated, and the results are controversial, ${ }^{7-9} \mathrm{E} 4$ may be related to the acceleration of cerebral atherosclerosis. It has been well established that the possession of $\varepsilon 4$, the allele for the $\mathrm{E} 4$ isoform, is a strong genetic risk factor for Alzheimer's disease and the presence and dose of the allele are positively correlated to the incidence of Alzheimer's disease. ${ }^{10-12}$ Therefore, the presence of the $\varepsilon 4$ allele may constitute a risk for cerebral white matter lesions through acceleration of atherosclerosis on the one hand, and may cause white matter lesions through a positive pathogenetic correlation with Alzheimer's disease on the other.

To examine this hypothesis, we investigated the association of the apoE genotype with the presence and grades of the cerebral white matter lesions in Alzheimer's disease. Furthermore, we evaluated the association of the presence of cerebral white matter lesions with several factors such as sex, age, and vascular risk factors, including hypertension, diabetes mellitus, and hyperlipidaemia.

Subjects and methods

Fifty five patients with Alzheimer's disease and 66 sex and age matched healthy control subjects were randomly selected from outpatients. The diagnosis of dementia and 
Table 1 Relation between factors and white matter lesions in Alzheimer's disease

\begin{tabular}{lllll}
\hline $\begin{array}{l}\text { White matter } \\
\text { lesion }\end{array}$ & $N$ & Age (mean (SEM) & e4 Allele (\%) & Female (\%) \\
\hline Grade 1 & 19 & $71.1(2.3) \mathrm{p}=0.0103^{\star}$ & $36.8 \mathrm{p}=0.6442 \dagger$ & $47.4 \mathrm{p}=0.047 \dagger$ \\
Grade 2 & 29 & $77.8(1.4)$ & 44.8 & 69.0 \\
Grade 3+4 & 7 & $80.6(2.3)$ & 14.3 & 85.7
\end{tabular}

^By one way ANOVA.

†By non-parametric Mann-Whitney test.

Grade 1=no lesions; grade $2=$ patchy lesions; grade 3=grade $2+$ confluent lesions; grade $4=$ grade 3+diffuse lesions.

Table 2 ApoE and white matter lesion in Alzheimer's disease (AD)

\begin{tabular}{lllllll}
\hline \multirow{5}{*}{ MRI } & \multicolumn{5}{c}{ Allele frequency } & \\
\cline { 3 - 5 } & N (allele) & $\varepsilon 2$ & $\varepsilon 3$ & $\varepsilon 4$ & & \\
\hline AD without lesions & 38 & 0.026 & 0.737 & 0.237 & $\mathrm{p}<0.01^{\star}$ & $\mathrm{NS}+$ \\
AD with lesions & 72 & 0.000 & 0.778 & 0.222 & $\mathrm{p}<0.001^{\star}$ & \\
Control & 132 & 0.038 & 0.909 & 0.053 & & \\
\hline
\end{tabular}

*Compared with control by $\chi^{2}$ test.

†Compared with $\mathrm{AD}$ without lesions by $\chi^{2}$ test.

Alzheimer's disease was made according to the DSM-IIIR criteria and those of the National Institute of Neurological and Cognitive Disorders Association, respectively. We determined the apoE genotype by the procedure described previously. ${ }^{13}$ Leucocyte DNA was extracted from the subjects' blood samples using standard phenol extracts. The DNA was amplified using the polymerase chain reaction with the specific primers, forward (5'-AGACAGTCT CCCTCTTGCTGA-3') and reverse (5'-CC TCCTTTCCTGACCCTGTCCTT-3'). The amplified DNA was cleaved with the restriction enzyme Hha I, and then analysed by Southern blotting.

The white matter lesions found by T2 weighted MRI (1.5 tesla) were evaluated in all 55 patients with Alzheimer's disease. The lesions were graded on the following four class scale: grade 1 (no lesions), grade 2 (punctate lesions), grade 3 (lesions reaching confluence), and grade 4 (confluent or irregular periventricular hyperintensity), according to the procedure described by Schmidt et al. ${ }^{14}$ The evaluation of MRI was blind to other information about the patients.

A patient was considered to have hypertension if the history contained one of the following: treatment for hypertension, medication with antihypertensive drugs, or a systolic/ diastolic blood pressure higher than 150/90 $\mathrm{mm} \mathrm{Hg}$, disclosed by at least three measurements. Patients were considered to have diabetes mellitus if they were taking medication (sulfonyl urea or insulin therapy), or if the blood sugar was higher than $180 \mathrm{mg} / \mathrm{dl}$ at any sampling. A patient was considered to have hyperlipidaemia if: they were presently being treated with antihyperlipidaemia drugs, or if the plasma total cholesterol was higher than $240 \mathrm{mg} / \mathrm{dl}$ or the triglyceride was higher than $180 \mathrm{mg} / \mathrm{dl}$ from at least two samples of fasting blood.

Firstly, the association of apoE genotype with the presence of white matter lesions was investigated and statistically examined using the $\chi^{2}$ test. Then the odds ratio (OR) of various factors, including possession of the $\varepsilon 4$ allele,
Table 3 Odds ratio of factors for white matter lesions in Alzheimer's disease

\begin{tabular}{lll}
\hline & $\begin{array}{l}\text { Odds ratio for white } \\
\text { matter lesions }\end{array}$ & p Value \\
\hline ApoE $\varepsilon 4$ allele & 1.09 & $>0.99$ \\
Age $>70$ & 7.20 & 0.006 \\
Age $>80$ & 4.27 & 0.041 \\
Female & 2.89 & 0.084 \\
Hypertension & 2.10 & 0.227 \\
Diabetes & 2.13 & 0.468 \\
Hyperlipidaemia & 0.92 & $>0.99$ \\
\hline
\end{tabular}

$\mathrm{p}$ Values by Fisher's exact test.

hypertension, diabetes, and hyperlipidaemia to the presence of the lesions was determined.

The association of factors was examined by Fisher's exact test using a $2 \times 2$ contingency table. The relation between the lesion grades and these factors was examined by the non-parametric Mann-Whitney's test. The relation between age and the lesion grades was examined by the non-parametric Spearman's rank correlation test.

\section{Results}

Cerebral white matter lesions were detected in $36(65.4 \%)$ of the 55 patients with Alzheimer's disease. Twenty nine patients $(44.8 \%)$ had patchy lesions, but neither confluent nor diffuse lesions in the white matter. Confluent or diffuse lesions were seen in seven of 55 patients $(14.3 \%)$. The mean age (SD) of the patients with patchy lesions and diffuse or confluent lesions was 77.8 (1.4) years old and 80.6 (2.3) years old, respectively, both of which were significantly higher than that of patients without lesions (71.1 (2.3) years old). The frequency of the possession of the $\varepsilon 4$ allele was $36.8 \%$ in patients without lesions, $44.8 \%$ in those with patchy lesions, and $14.3 \%$ in those with confluent or diffuse lesions. There was no statistically significant relation between the $\varepsilon 4$ allele frequency and the lesion grades $(p=0.642)$. The female proportion of patients without white matter lesions was $47.6 \%$, whereas that of the patients with patchy and confluent or diffuse lesions was $69.0 \%$ and $85.7 \%$, respectively. There was a significant positive relation between the female proportion and the lesion grades $(\mathrm{p}=0.047$, table 1$)$.

In the control subjects, the frequency of the $\varepsilon 4$ allele was 0.053 , which is consistent with previous studies in the Japanese population. ${ }^{11}$ The allele frequency was 0.237 and 0.222 in the groups of patients with Alzheimer's disease with and without white matter lesions, respectively, and was significantly higher than that in the control subjects. However, the allele frequency was not significantly different in patients with Alzheimer's disease but without white matter lesions ( $\mathrm{p}=0.986$, table 2). Furthermore, the OR for the possession of the $\varepsilon 4$ allele to the presence of white matter lesions was 1.09 so that the possession of the $\varepsilon 4$ allele was not a risk for the presence of white matter lesions (table 3).

By contrast with the apoE genotype, aging was a significant risk for the presence of white matter lesions. The OR of ages older than 70 years and older than 80 years was 7.20 $(\mathrm{p}=0.006)$ and $4.27(\mathrm{p}=0.041)$, respectively. As 
mentioned above, the proportion of women was significantly higher in the group with white matter lesions, and the OR for women was 2.89 $(p=0.084)$. The ORs of the other vascular risk factors, the presence of hypertension, diabetes mellitus, and hyperlipidaemia, were low and not significant risk factors for the lesions (table 3).

\section{Discussion}

Although the pathogenetic mechanism causing cerebral white matter lesions has not been resolved, the deep cerebral white matter is vulnerable to chronic ischaemia, because arteries from the leptomeningeal border zones to the deep white matter, and medullary arterioles must pass through this region. The periventricular white matter is thought to be particularly susceptible to ischaemia because the arteries irrigating these areas have few anastomoses. Therefore, chronic ischaemic damage to the white matter could cause white matter lesions. The possession of the $\varepsilon 4$ allele has been disclosed as a significant vascular risk factor, because homozygous $\varepsilon 4$ alleles are closely related to high total and LDL cholesterol, ${ }^{5}$ and are a significant risk for fatal myocardial infarction. ${ }^{15}$ However, the relation between the $\varepsilon 4$ allele and cerebral vascular lesions is controversial. $^{7-9}$

Cerebral amyloid angiopathy is associated with ischaemic white matter lesions in Alzheimer's disease, ${ }^{16}$ and it has been shown that the extent of the cerebral amyloid angiopathy pathology is related to the apoE genotype. ${ }^{17}$ Although the presence of white matter lesions is not related to the apoE genotype in Dutch hereditary amyloid angiopathy, ${ }^{18}$ the relation between the presence of white matter lesions and cerebral amyloid angiopathy in Alzheimer's disease has not been elucidated. The present study showed no association between the apoE genotype and white matter lesions in Alzheimer's disease and suggests that there is no correlation between white matter infarction and the extent of amyloid angiopathy; however, further studies are needed to show the relation between white matter lesions and the extent of amyloid angiopathy in Alzheimer's disease. The present study demonstrated that aging was a significant risk for white matter lesions in Alzheimer's disease, consistent with previous studies of Alzheimer's disease. ${ }^{19}$ In normal brains, however, there is a significant negative correlation between white matter volume and age but no significant association between white matter hyperintensity area, shown by MRI, and age. ${ }^{20}$ Hypotension as a result of postural changes may produce a reduction in cerebral blood flow and can cause repetitive ischaemia in the cerebral white matter, especially in elderly patients. ${ }^{21}$ Moreover, changes in vascular structure may provide this association. In addition to arteriosclerotic changes, the collagenosis of veins in the periventricular white matter is associated with aging and the degree of white matter lesions. ${ }^{22}$ Compared with normal brains, these changes in vascular structure may be enhanced in the brains of patients with Alzheimer's disease.
Another significant risk factor for the presence of white matter lesions is sex-that is, a predominance of women, consistent with previous research. ${ }^{123}$ The reason for female predominance is not the age difference between the sexes because there was no significant difference in age between the men and women in the present study. In a community based study, there was no significant difference in the frequency of ischaemic white matter lesions between men and women. ${ }^{24}$ Recent epidemiological studies have indicated that women have a higher risk of the disease, especially at ages older than $80 .^{25}{ }^{26}$ The decline in estrogen has been reported to be related to the risk of Alzheimer's disease in postmenopausal women, and replacement of estrogen reduces the morbidity of the disease. Estrogens have protective effects against neuronal death in in vitro studies. ${ }^{27} 29$ Therefore, the estrogen decline in menopausal women may be related to the degenerative damage in the cerebral white matter, although further studies are needed to elucidate the effect of sex difference on the presence of white matter lesions.

In the present study, there was no association between $\varepsilon 4$ allele frequency and grade of white matter lesion, and lesion grade increased with age and in women. The reason of no association between apoE genotype and white matter lesions in Alzheimer's disease is still undetermined; however, the white matter lesions may be caused by changes in small vessels, which could be affected by age rather than lipid metabolism. Therefore, an age adjusted study would be needed to elucidate the relation of $\varepsilon 4$ and white matter lesions; this would be difficult to perform in the current study because of its small size and statistical power issues.

1 Diaz JF, Merskey H, Hachinski VC, et al. Improved recognition of leukoaraiosis and cognitive impairment in Alzheimer's disease. Arch Neurol 1991;48:1022-5.

2 Brun A, Englund E. A white matter disorder in dementia of the Alzheimer type: a pathoanatomical study. Ann Neurol

3 Pantoni L, Garcia JH. Pathogenesis of leukoaraiosis: a review. Stroke 1997;28:652-9.

4 Lehtimaki T, Moilanen T, Viikari J, et al. Apolipoprotein E phenotypes in Finnish youths: a cross-sectional and 6-year follow-up study. 7 Lipid Res 1990;31:487-95.

5 Eichner JE, Kuller LH, Orchard TJ, et al. Relation of apolipoprotein $\mathrm{E}$ phenotype to myocardial infarction and mortality from coronary artery disease. Am $\mathcal{F}$ Cardiol 1993; 71:160-5.

6 van Bockxmeer FM, Mamotte CD. Apolipoprotein $\varepsilon 4$ homozygosity in young men with coronary heart disease. Lancet 1992;340:879-80.

7 Pedro Botet J, Senti M, Nogues X, et al. Lipoprotein and apolipoprotein profile in men with ischemic stroke. Role of apolipoprotein profile in men with ischemic stroke. Role of

8 Couderc R, Mahieux F, Bailleul S, et al. Prevalence of apolipoprotein $\mathrm{E}$ phenotypes in ischemic cerebrovascular poprotein E phenotypes in ischemic cerebrovascu
disease. A case-control study. Stroke 1993;24:661-4.

9 Basun H, Corder EH, Guo Z, et al. Apolipoprotein E polymorphism and stroke in a population sample aged 75 years or more. Stroke 1996;27:1310-5.

10 Corder EH, Saunders AM, Strittmatter WJ, et al. Gene dose of apolipoprotein E type 4 allele and the risk of Alzheimer's disease in late onset families. Science 1993;261:921-3.

11 Kawamata J, Tanaka S, Shimohama S, et al. Apolipoprotein E polymorphism in Japanese patients with Alzheimer's disease or vascular dementia. $\mathcal{J}$ Neurol Neurosurg Psychiatry 1994;57:1414-6.

12 Saunders AM, Hulette O, Welsh Bohmer KA, et al. Specificity, sensitivity, and predictive value of apolipoprotein-E genotyping for sporadic Alzheimer's disease. Lancet 1996; 348:90-3.

13 Toji H, Kawakami H, Kawarai T, et al. No association between apolipoprotein $\mathrm{E}$ alleles and olivopontocerebellar atrophy. F Neurol Sci 1998;158:110-2. 
14 Schmidt R, Fazekas F, Kleinert G, et al. Magnetic resonance imaging signal hyperintensities in the deep and subcortical white matter. A comparative study between stroke patients and normal volunteers. Arch Neurol 1992;49:825-7.

15 Lehtinen S, Lehtimaki T, Sisto T, et al Apolipoprotein $\mathrm{E}$ polymorphism, serum lipids, myocardial infarction and severity of angiographically verified coronary artery disease in men and women. Atherosclerosis 1995;114:83-9

16 Olichney JM, Hansen LA, Hofstetter CR, et al. Cerebral infarction in Alzheimer's disease is associated with severe amyloid angiopathy and hypertension. Arch Neurol 1995; 52:702-8.

17 Premkumar DR, Cohen DL, Hedera P, et al. Apolipoprotein E- $\varepsilon 4$ alleles in cerebral amyloid angiopathy and cerebrovascular pathology associated with Alzheimer's disease. $A m \mathcal{F}$ Pathol 1996;148:2083-95.

18 Bornebroek M, Haan J, Van Duinen SG, et al. Dutch hereditary cerebral amyloid angiopathy: structural lesions and tary cerebral amyloid angiopathy: structural lesions

19 Brilliant M, Hughes L, Anderson D, et al. Rarefied white matter in patients with Alzheimer disease. Alzheimer Dis matter in patients with A

20 Guttmann CRC, Jolesz FA, Kikinis R, et al. White matter changes with normal aging. Neurology 1998;50:972-8.

21 Matsubayashi K, Okumiya K, Wada T, et al. Postural dysregulation in systolic blood pressure is associated with worsened scoring on neurobehavioral function tests and leukoaraiosis in the older elderly living in a community. Stroke 1997;28:2169-73.
22 Moody DM, Brown WR, Challa VR, et al. Cerebral microvascular alterations in aging, leukoaraiosis, and Alzheimer's disease. Ann N Y Acad Sci 1997;826:103-16.

23 Henon H, Godefroy O, Lucas C, et al. Risk factors and leuoaraiosis in stroke patients. Acta Neurol Scand 1996;94: 137-44.

24 Jorgensen HS, Nakayama H, Raaschou HO, et al. Leukoaraiosis in stroke patients. The Copenhagen stroke study. Stroke 1995;26:588-92.

25 Launer LJ, Andersen K, Dewey ME, et al. Rates and risk factors for dementia and Alzheimer's disease: results from EURODEM pooled analyses. EURODEM Incidence Research Group and Work Groups. European studies of Research Group and Work Groups.
dementia. Neurology 1999;52:78-84.

26 Letenneur L, Gilleron V, Commenges D, et al. Are sex and educational level independent predictors of dementia and Alzheimer's disease? Incidence data from the PAQUID project. F Neurol Neurosurg Psychiatry 1999;66:177-83.

27 Gridley KE, Green PS, Simpkins JW. A novel, synergistic interaction between $17 \beta$-estradiol and glutathione in the protection of neurons against $\beta$-amyloid 25-35-induced protection of neurons itro. Mol Pharmacol 1998;54:874-80.

28 Sawada H, Ibi M, Kihara T, et al. Estradiol protects mesencephalic dopaminergic neurons from oxidative stressinduced neuronal death. 7 Neurosci Res 1998;54:707-19.

29 Sawada H, Ibi M, Kihara T, et al. Mechanisms of antiapoptotic effects of estrogens in nigral dopaminergic neurons. FASEB $\mathcal{F} 2000$ (in press). 